\title{
The progression of $\mathrm{CD56}^{+}$myeloid sarcoma: A case report and literature review
}

\author{
XIN WANG ${ }^{1}$, WEN-SHENG LI $^{2}$, YAN ZHENG ${ }^{1}$, ZHAO-XIA YING ${ }^{1}$, YONG-XIAN WANG ${ }^{1}$, \\ YING-MEI WANG ${ }^{3}$, JUN-FENG ZHENG ${ }^{3}$ and SHENG-XIANG XIAO ${ }^{1}$
}

${ }^{1}$ Department of Dermatology, The Second Affiliated Hospital of Xi'an Jiaotong University, Xi'an, Shaanxi 710004;
${ }^{2}$ Department of Pathology, The Third Affiliated Hospital of Xi'an Jiaotong University, Xi'an, Shaanxi 710068;
${ }^{3}$ Department of Pathology, Xijing Hospital, Fourth Military Medical University, Xi'an, Shaanxi 710032, P.R. China

Received December 5, 2014; Accepted December 23, 2015

DOI: $10.3892 / \mathrm{ol} .2016 .4352$

\begin{abstract}
The current study presents a case of cluster of differentiation $(\mathrm{CD}) 56^{+}$myeloid sarcoma in a patient that initially presented with skin lesions, and provides evidence for the clinical and differential diagnosis of myeloid sarcoma. The patient of the present case report was a 65 -year-old man who was admitted to hospital with a six-month history of bilateral purple-red papules and nodules, which were present on the upper limbs of the patient and had spread over his whole body one month prior to admission to the hospital. Pathological examination demonstrated a diffuse infusion of primitive round cells at the papillary dermis and subcutaneous tissues. The infiltrated cells were 40-60 $\mu \mathrm{m}$ in diameter and morphologically identical. Immunohistochemical examination revealed that the cells expressed myeloperoxidase, CD56, CD43 and T-cell intracytoplasmic antigen. In addition, several cells expressed CD34, and 90\% of the cells expressed Ki67. While the majority of cells in myeloid sarcoma do not express CD56, the present case was a myeloid sarcoma that expressed CD56, which is extremely rare. The sarcoma in the present patient progressed rapidly, and the patient died eight months following the onset of disease. Clinicians should be aware of $\mathrm{CD}^{+} 6^{+}$myeloid sarcoma, which is easily misdiagnosed and inappropriately treated. Consequently, myeloid sarcoma may have a high malignancy and poor outcome for patients.
\end{abstract}

\section{Introduction}

Myeloid sarcoma is a form of solid tumor, and is composed of immature white blood cells known as myeloblasts $(1,2)$.

Correspondence to: Dr Yan Zheng, Department of Dermatology, The Second Affiliated Hospital of Xi'an Jiaotong University, 157 West 5th Road, Xi'an, Shaanxi 710004, P.R. China

E-mail: zenyan66@126.com

Key words: myeloid sarcoma, CD56, Ki67, granulocytic sarcoma, immunohistochemistry
The disease is classified into four groups according to tumor cell type, namely granulocytic sarcoma, primitive monocytic sarcoma, myeloid sarcoma of hematopoietic cell and primary myeloid sarcoma without the presentation of other hematological diseases $(1,2)$. Myeloid sarcoma may develop at any age, but occurs most commonly in children and young individuals (3). The incidence of myeloid sarcoma in females is slightly higher than in males, with a ratio of 1.42:1 (3) The proportion of patients with acute myelocytic leukemia (AML) accompanied with myeloid sarcoma is $2-8 \%$, and $\sim 10 \%$ of type M2 AML cases develop into myeloid sarcoma $(1,4)$. Genetic or chromosomal abnormalities are associated with the disease (5), and factors including malnutrition, cellular immune dysfunction, increased white blood cells (3), and myeloblasts expressing T-cell surface markers, cluster of differentiation (CD)13, or CD14 (6) serve as risk factors. The mortality rate of patients is extremely high, and in a study of 23 non-leukemic myeloid sarcoma cases, it was observed that the average progression free survival was 12.5 months, the average survival rate was 32.9 months and the expected 3 -year survival rate was $41 \%$ (7).

No universally accepted treatment for the disease is currently available. For myeloid sarcoma other than leukemia, surgical removal of the tumor followed by local radiotherapy may be performed. In certain cases, myeloid sarcoma is accompanied by other hematological diseases, in which case systemic chemotherapy or combined treatment of surgery, local radiotherapy and systemic chemotherapy may be performed (8) In the majority of cases, myeloid sarcomas are accompanied by other hematological diseases, including AML, chronic myeloproliferative diseases and myelodysplastic syndrome. However, Kohli et al (9) reported a case in which myeloid sarcoma occurred without developing acute myeloid leukaemia or other hematological diseases, it only formed multiple metastatic deposits. The patient received systemic chemotherapy in addition to radiotherapy, due to the limited therapeutic effect of chemotherapy alone (9).

Although myeloid sarcoma is able to develop in the absence of other systemic diseases, it is a complicated disease with high mortality and low survival rates in itself, and patients presenting with features of the disease should receive combined treatment as soon as possible. 


\section{Case report}

A 65-year-old male patient was admitted to The Second Affiliated Hospital of Xi'an Jiaotong University (Xi'an, China) on November 28, 2013, with a 6-month history of bilateral purple-red papules and nodules on the upper limbs, which had spread over the whole body of the patient one month prior to admission to the hospital (Fig. 1A). The purple-red papules and nodules measured between $3.0 \mathrm{~mm}$ and $1.0 \mathrm{~cm}$ in size, and had appeared bilaterally on the upper limbs six months prior to admission to the hospital, with no apparent identifiable cause. A proportion of the papules and nodules were observed to be merged together, forming large nodules with clear borders and a hard texture. Generally, there was no mobility of the papules and nodules. Since the patient experienced no other symptoms, with the exception of slight itching, the skin lesions were not considered to be of serious concern, even though the number of the papules and nodules had increased and spread to the whole body with no apparent cause. The patient was admitted to The Second Affiliated Hospital of Xi'an Jiaotong University for a skin biopsy of the left elbow. Following the biopsy, the skin samples were stained with hematoxylin and eosin (catalog no., AR1180-100; Wuhan Boster Biological Technology, Ltd., Wuhan, China). The patient refused to undergo an immunohistochemical examination, due to the relatively high cost associated with the test.

One of the skin lesions of the patient became aggravated 10 days following admission to the hospital, without any apparent cause. The patient was then admitted to a local hospital, where was diagnosed with Sjogren's syndrome. The patient was provided with oral drugs, the name and dose of which are not known. However, the effects of the treatment were limited, and the lesion progressed. The patient experienced pain in his throat and the sensation of a foreign body three days after treatment. The patient also had dysphagia and did not eat or drink water for three days. In consequence, the patient returned to The Second Affiliated Hospital of Xi'an Jiaotong University for additional diagnosis and treatment. The patient's throat was assessed by the naked eye with a mirror and a laryngoscope, and abnormal parenchyma were identified. Therefore, the tumor was speculated to have not only invaded the throat, but to have also invaded the esophagus and other digestive organs. Although the patient was conscious following the onset of the disease, his mental state was poor. In addition, the patient had not defecated for three days, although urinary function was normal according to a 24-hour urine volume test. The weight of the patient had decreased by $4.5 \mathrm{~kg}$, compared with the weight recorded during his visit to the hospital one month earlier. The patient had no fever, infection, anemia or joint pain, but had a history of hypertension and cerebral infarction, and was suffering from hemiplegia.

Physical examination of the patient's temperature, respiration, pulse and blood pressure demonstrated that the vital signs of the patient were stable. However, the patient had difficulty closing his eyes. Hemiplegic gait and swollen lymph nodes behind the right ear were also observed, which were painful when pressed. In addition, nodules of $\sim 1.0 \mathrm{~cm}$ in size were observed on the throat. Examination of the skin revealed the presence of red papules and nodules that measured between $3.0 \mathrm{~mm}$ and $2.5 \mathrm{~cm}$ in size, which had spread over the body.
The distribution of the papules and nodules were diffuse or focused, and the shape of the papules and nodules were round or oval. The nodules protruded above the skin surface, and their borders were defined, while their texture was hard. The surface of certain papules and nodules had burst. Several papules and nodules had merged together to form irregular nodules. The nodules at the bilateral zygomatic regions and cheeks were observed to be merged together, with pitting surface edema. A red nodule of $\sim 1.0 \mathrm{~cm}$ in size was observed at each of the palpebral conjunctiva of the patient. Sporadic purple-red petechia or bruising was also observed at the flexor side of the bilateral lower limbs, with defined margins, and the color did not disappear when pressure was applied (Fig. 1B-H).

Laboratory examinations revealed that the neutrophil ratio of the patient was $72.24 \%$ (normal range, 51-75\%), while the monocyte ratio was $2.74 \%$ (normal range, $3-8 \%$ ). On chest X-ray (SOMATOM Definition AS 64, Siemens AG, Munich, Germany), increased bronchovascular shadows on the lung were observed. Computed tomography (LightSpeed VCT XT; GE Healthcare Life Sciences, Chalfont, UK) also revealed increased bronchovascular shadows on the lung, and bilateral cord-like lesions at the apex of the lung (Fig. 2A and B).

Histopathological examination revealed that the epidermis was not involved, and the lesions were primarily focused on the papillary dermis and subcutaneous tissues. There was diffuse infiltration of round, medium-sized, primitive cells. The majority of the infiltrating cells were morphologically identical, and compared with the mature cells, the ratio of cytoplasm to nuclear volume in infiltrating cells was decreased and the nuclei of infiltrating cells was increased. The nuclei of the cells were primarily round or oval, although lobate or kidney-shaped nuclei were also observed in several cells. The cells had delicate chromatin and small nucleoli, and numerous cells undergoing mitosis were observed (Fig. 2C-E).

Immunohistochemical examination was performed using the following antibodies: Monoclonal mouse anti-human CD56 (dilution, 1:800; catalog no., SC-106; Santa Cruz Biotechnology, Inc., Dallas, TX, USA), monoclonal rabbit anti-human CD43 (dilution, 1:1,000; catalog no., SAB5500067), monoclonal mouse anti-human CD34 (dilution, 1:1,000; catalog no., SAB4700736), monoclonal mouse anti-human Ki67 (dilution, 1:800; catalog no., P6834) (Sigma-Aldrich, St. Louis, MO, USA), polyclonal goat anti-human T-cell intracytoplasmic antigen (TIA-1; dilution, 1:1,000; catalog no., SC-1751; Santa Cruz Biotechnology, Inc.), monoclonal rabbit anti-human CD3 (dilution, 1:600; catalog no., SAB5500057), monoclonal rabbit anti-human CD4 (dilution, 1:600; catalog no., SAB5500064), monoclonal rabbit anti-human CD7 (dilution, 1:600; catalog no., SAB5500071), monoclonal rabbit anti-human CD20 (dilution, 1:1,000; catalog no., SAB5500049) (Sigma-Aldrich), polyclonal rabbit anti-human CD123 (dilution, 1:800; catalog no., SC-681; Santa Cruz Biotechnology, Inc.), monoclonal rabbit anti-human paired box 5 (PAX-5; dilution, 1:800; catalog no., SAB5500160), polyclonal rabbit anti-human granzyme B (dilution, 1:800; catalog no., HPA003418) and monoclonal rabbit anti-human myeloperoxidase (MPO; dilution, 1:1,000; catalog no., HPA021147) (Sigma-Aldrich). The antibodies were diluted in $5 \%$ bovine serum albumin, and the tissues were incubated overnight at $4^{\circ} \mathrm{C}$. DBA color developing reagent was used to reveal the staining, and the slides were 


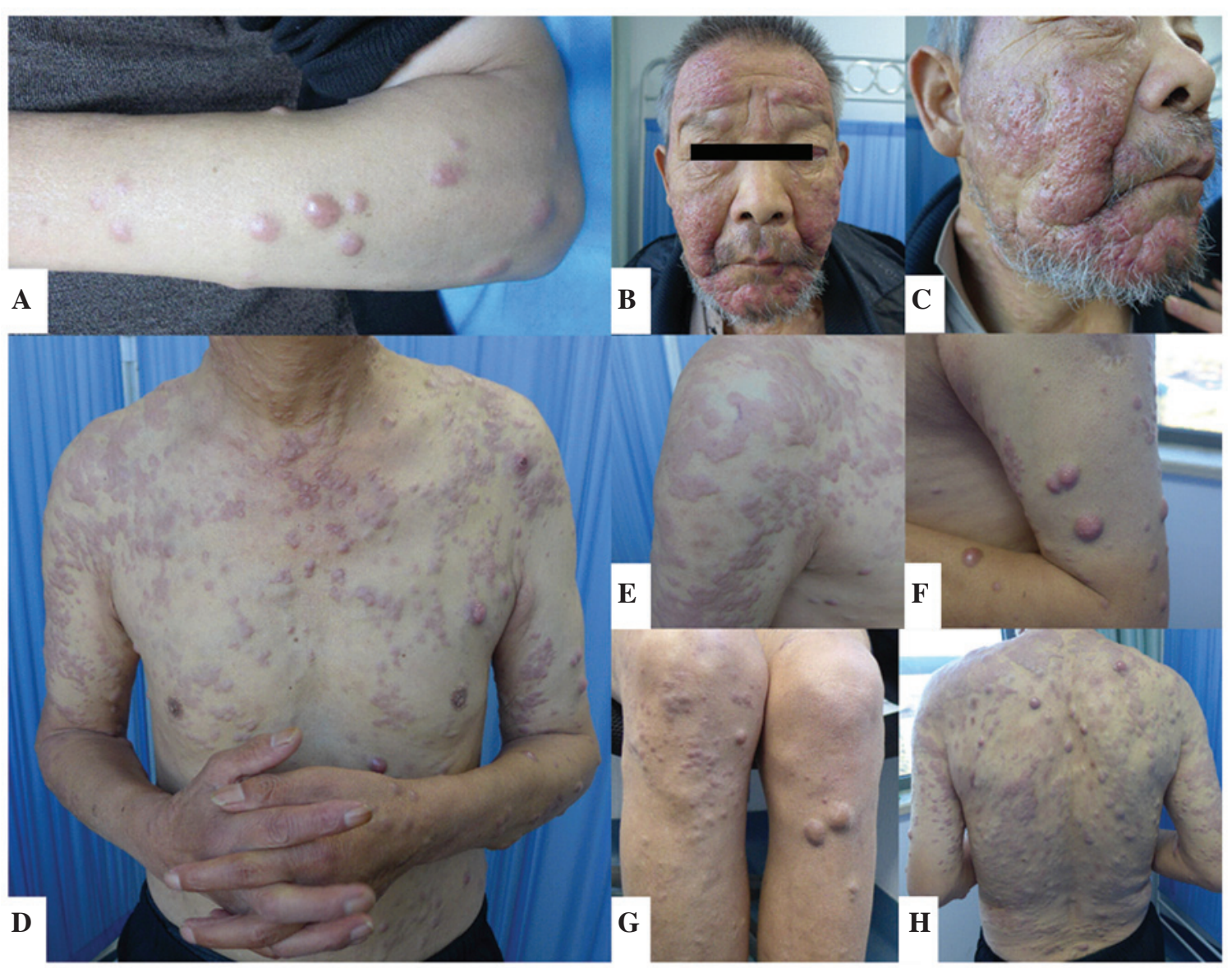

Figure 1. Clinical presentation of the myeloid sarcoma in the patient of the present case report. (A) Purple-red papules and nodules measuring between 3.0 mm and $1.0 \mathrm{~cm}$ in size were present on the upper limbs of the patient. (B and C) Red nodules at the bilateral zygomatic regions and cheeks were merged together. A red nodule of $\sim 1.0 \mathrm{~cm}$ in size was observed at each palpebral conjunctiva. (D-H) Round and oval red papules and nodules measuring between 3.0 mm and $2.5 \mathrm{~cm}$ in size were observed over the whole body of the patient. Several papules and nodules were merged together to form irregular nodules, which protruded above the skin surface and had clear borders. The surface of certain papules and nodules had burst.

subsequently analyzed through a microscope (CX21; Olympus Corporation, Tokyo, Japan) aided by NanoZoomer 2.0-HT (Hamamatsu Photonics, Hamamatsu, Japan) and Image Pro Plus 6.0 (Media Cybernetics, Inc., Rockville, MD, USA). The results demonstrated that all the cells expressed (MPO), CD56, CD43 and TIA-1 (Fig. 3). In total, 70\% of the cells also expressed CD34, and $90 \%$ of the cells expressed Ki67. However, the cells did not express CD3, CD4, CD7, CD123, CD20, PAX-5, granzyme B or Epstein-Barr virus. In consequence, the patient was diagnosed with myeloid sarcoma. The patient refused to be hospitalized for treatment due to the relatively high cost. Therefore, no treatment was administered to the patient. The patient died three days later due to a throat blockage, which prevented the patient from eating or drinking water resulting in hypoxia and malnourishment. The course of the disease was eight months from the initial presentation of symptoms. Written informed consent was obtained from the patient for the publication of the present study and use of accompanying images.

\section{Discussion}

Myeloid sarcoma is composed of primitive and immature cells, which originate, mature and differentiate in the bone marrow (1). Myeloid sarcoma is classified into four subtypes according to the various cell types involved, as follows: i) Granulocytic sarcoma; ii) primitive monocytic sarcoma; iii) myeloid sarcoma of hematopoietic cells; and iv) primary myeloid sarcoma without the presentation of other hematological diseases $(1,2)$.

Previous studies have suggested that genetic or chromosome abnormalities are associated with myeloid sarcoma (10). Several gene rearrangements and the presence of three copies of the chromosome 8 are relatively common chromosome abnormalities observed in myeloid sarcoma (1). Malnutrition, cellular immune dysfunction and increased levels of white blood cells are also risk factors for myeloid sarcoma (3). Gastrointestinal tract, ovary, testes, skeletal muscle, nervous tissue, normal brain cells and natural killer (NK) cells express CD56 (11). In addition, cells, tissues and tumors generated from the ectoderm, as well as tumors generated from the mesoderm and NK/T-cell lymphoma also express CD56 (12). Previous immunohistochemical examinations have revealed that the majority of patients with myeloid sarcoma do not express CD56 (12). However, this was not the case for the present patient. Previous studies have reported that certain chromosomal abnormalities such as $\mathrm{t}(8 ; 21)$, are often associated with CD56 expression, which increases the incidence of myeloid sarcoma or relapse of myeloid sarcoma following treatment (11). Myeloid sarcoma that expresses CD56 may be 


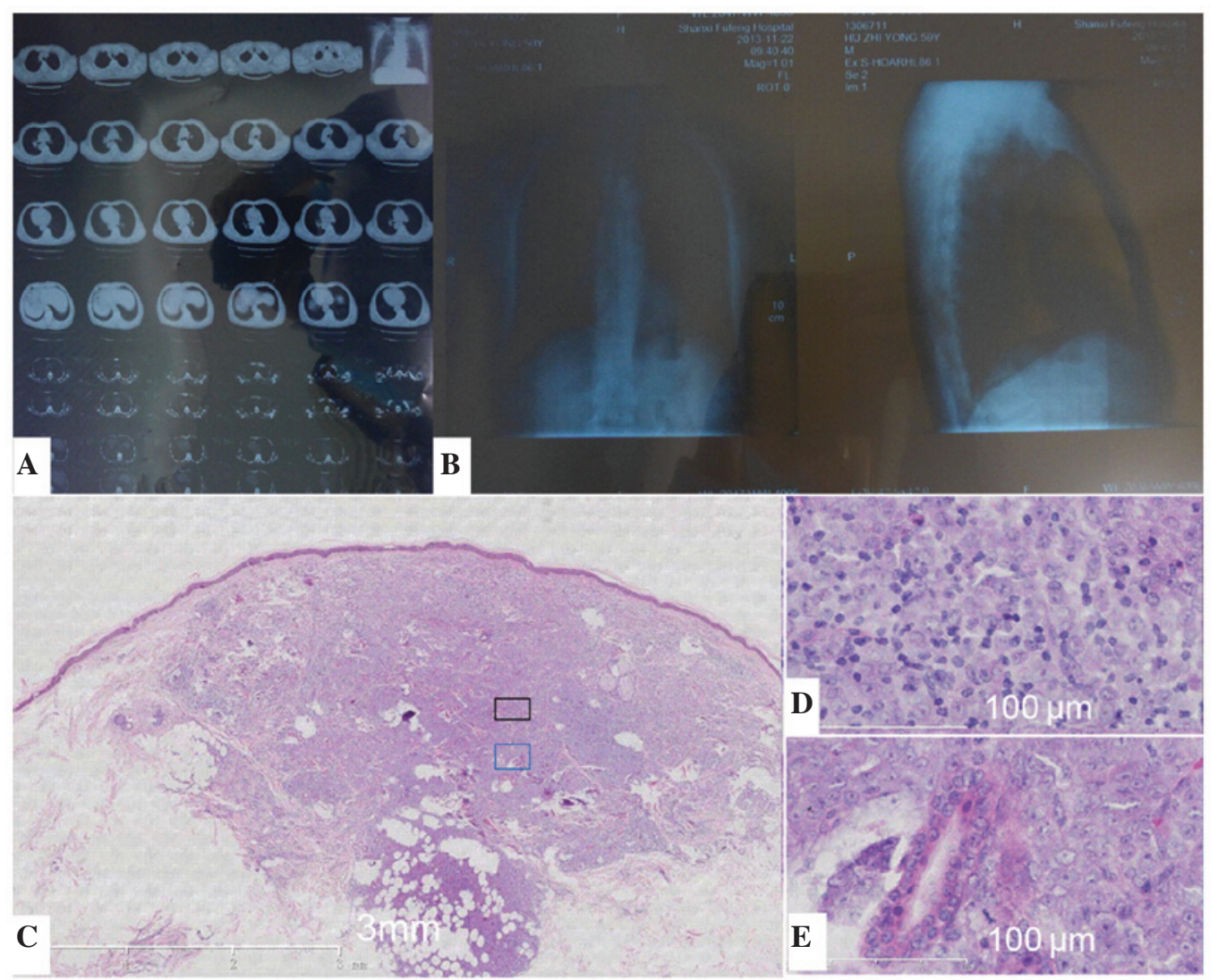

Figure 2. CT, X-ray and HE examination of the present patient with myeloid sarcoma. (A) CT revealed increased bronchovascular shadows on the lung and bilateral cord-like lesions at the apex of the lungs. (B) Chest X-ray revealed increased bronchovascular shadows on the whole lung. Left image, anteroposterior film; right image, lateral film. (C) A skin biopsy of the left elbow revealed by HE staining that the lesions were focused on the papillary dermis and subcutaneous tissues. (D and E) Diffuse infiltration of round, medium-sized (40-60 $\mu \mathrm{m}$ ), primitive cells was observed. Generally, the shape of the cells was identical, and the cell nuclei were primarily round or oval, although lobate or kidney-shaped nuclei were also observed in several cells. The cells had delicate chromatin and small nucleoli, and a large number of cells were undergoing mitosis. CT, computed tomography; HE, hematoxylin and eosin.
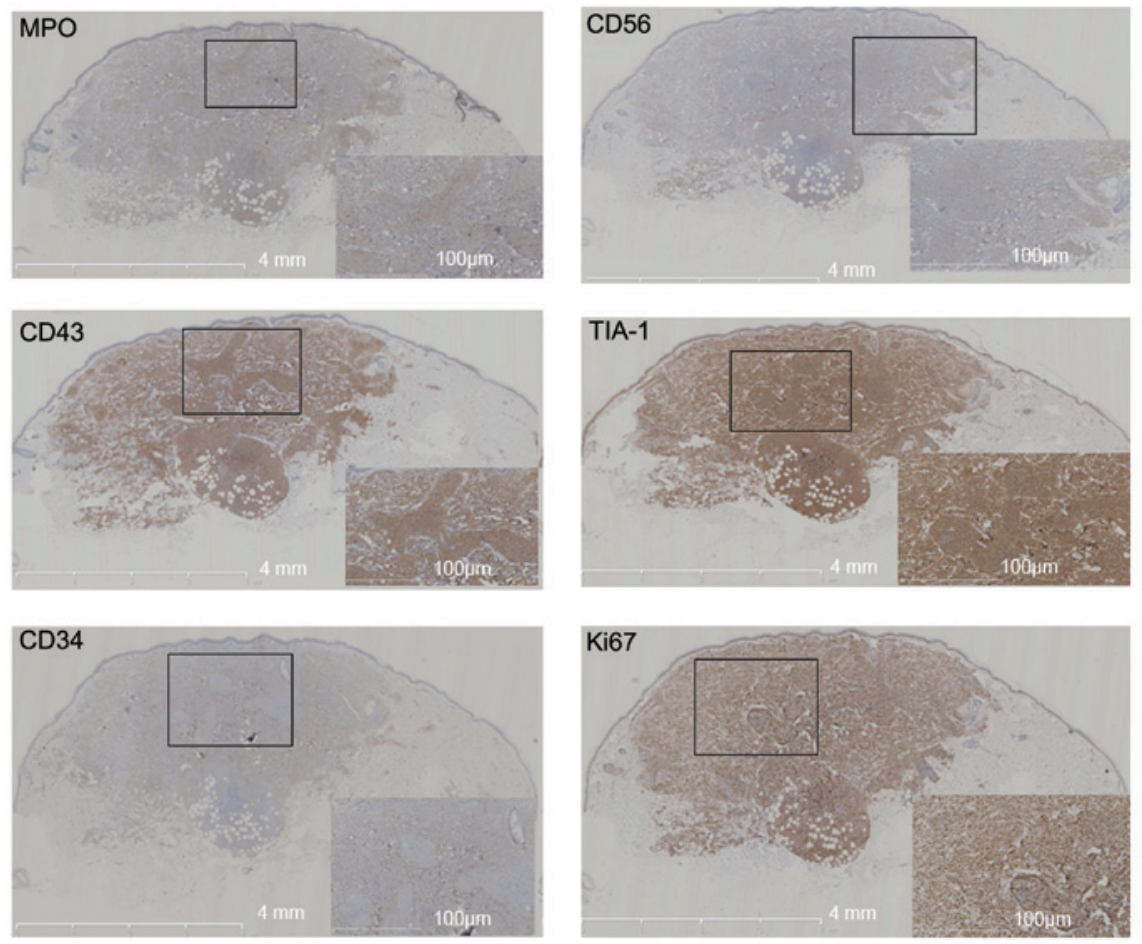

Figure 3. Immunohistochemical examination of the myeloid sarcoma corresponding to the patient of the present case report. All the cells expressed myeloperoxidase, cluster of differentiation (CD)56, CD43 and T-cell intracytoplasmic antigen. In addition, 70\% of the cells expressed CD34, and $90 \%$ of the cells expressed Ki67. MPO, myeloperoxidase; TIA-1, T-cell intracytoplasmic antigen. 
associated with a poor prognosis, which was confirmed by the outcome observed in the present patient. In addition, $90 \%$ of the cells in the myeloid sarcoma of the present patient expressed Ki67, which is also known to lead to a poor prognosis (7).

The clinical symptoms of myeloid sarcoma are determined by the tumor features, which are primarily characterized by dysfunction or functional disorders of the tissues or organs that are affected (13). The immunophenotypic features of myeloid sarcoma include expression of CD68, MPO, CD13, CD33, CD117 and CD43; and no expression of CD3, CD20 or CD7 (12,14-16). In certain patients, the expression of CD34 and terminal deoxynucleotidyl transferase (TdT) has been reported (17). However, the majority of patients with myeloid sarcoma do not express CD56, with the exception of rare examples, including the present patient (12).

The incidence of myeloid sarcoma is extremely low $(1,18)$; the exact data is not clear as the disease may be easily misdiagnosed as malignant lymphoma, metastatic cancer and other diseases $(8,19)$. However, the incidence of myeloid sarcoma in AML is 2-9\% (8). In certain cases, myeloid sarcoma is accompanied by hematological malignant tumors, and the symptoms may be similar to those of autoimmune diseases (20). Therefore, a differential diagnosis between myeloid sarcoma and lymphoblastic lymphoma, blastic plasmacytoid dendritic cell neoplasm and small-blue-round-cell tumor must be confirmed (10).

Lymphoblastic lymphoma may present with symptoms of skin invasion and MPO expression (21). Myeloid cell differentiation also distinguishes lymphoblastic lymphoma from myeloid sarcoma (12). Immunophenotypic features are extremely important for a differential diagnosis between lymphoblastic lymphoma and myeloid sarcoma (22). Thus, although myeloid sarcoma may express TdT, CD34, PAX-5, CD7 and CD10, these markers are not generally observed in myeloid sarcoma, but are frequently expressed in lymphoblastic lymphoma $(22,23)$.

Blastic plasmacytoid dendritic cell neoplasm is primarily observed in elderly male patients (24). The pathological features of this disease are similar to myeloid sarcoma (24). The morphological characteristics of the tumor cells are similar to myeloblasts or lymphocytes, but no blood vessels are identified in the tumor cells, which, by contrast, is frequently observed in myeloid sarcoma (24). In addition, inflammation or necrosis surrounding the tumor is also rare in blastic plasmacytoid dendritic cell neoplasm, but not in myeloid sarcoma (24). Tumor cells in blastic plasmacytoid dendritic cell neoplasm generally express CD4, but do not express MPO or TIA-1 (24).

Small-blue-round-cell tumors, including neuroblastoma, rhabdomyosarcoma and Ewing's sarcoma, are primarily observed in children, and generally do not express CD43 or MPO, which aids the differential diagnosis from myeloid sarcoma $(18,19)$.

There is no general treatment method for myeloid sarcoma currently available. For myeloid sarcomas other than leukemia, surgical treatment for the removal of the tumor, followed by local radiotherapy may be performed (8). For certain patients, where myeloid sarcomas are accompanied by other hematological diseases, systemic chemotherapy or combined treatment with surgical removal, local radiotherapy and systemic chemotherapy may be performed, depending on the particular situation of each individual patient (8). Although previous studies have reported the spontaneous remission of myeloid sarcomas (25), the majority of these were accompanied with other hematological diseases (18). Therefore, treatment is required to improve the survival rate of patients with myeloid sarcoma.

In the present study, the initial symptom of the elderly male patient was the presence of purple-red papules and nodules. The symptoms were not deemed to be too serious until the skin lesions spread to the entire body. A month following the initial admission to the hospital, the patient experienced throat pain with the sense of a foreign object and dysphagia, and was unable to ingest water and food for three days. Subsequently, the patient underwent additional diagnosis, and following examination, the presence of a tumor that had invaded the throat, esophagus and other digestive organs was observed. The progression of the myeloid sarcoma in the present patient was eight months between the initial development of the skin lesion and the date when the patient succumbed to the disease.

In conclusion, myeloid sarcoma may either occur alone or be accompanied by a hematological disease (19). In either case, it is a comprehensive disease, with rapid development and a poor prognosis. A synergistic method, which may be adapted according to the requirements of the patient and includes local or systemic chemotherapy and surgical treatment for the removal of the tumor, should be selected for treatment as soon as features of disease onset are identified (9). Future research on this disease is required in order to develop a more effective treatment.

\section{Acknowledgements}

The abstract of the present case report was presented at the Third Eastern Asia Dermatology Congress September 24-26, 2014 in Jeju, Korea, and was published by the Japanese Dermatological Association as abstract no. P-152 in Poster Abstracts, Wang X, Li WS, Zheng Y and Zheng JF: A case report of CD56+myeloid sarcoma: Natural history and literature review. J Dermatol 41 (Suppl 1): 35, 2014.

\section{References}

1. Di Veroli A, Micarelli A, Cefalo M, Ceresoli E, Nasso D, Cicconi L, Mauramati S, Ottaviani F, Venditti A and Amadori S: Recurrence of a $t(8 ; 21)$-positive acute myeloid leukemia in the form of a granulocytic sarcoma involving cranial bones: A diagnostic and therapeutic challenge. Case Rep Hematol 2013: 245395, 2013.

2. Aboutalebi A, Korman JB, Sohani AR, Hasserjian RP, Louissaint A Jr, Le L, Kraft S, Duncan LM and Nazarian RM: Aleukemic cutaneous myeloid sarcoma. J Cutan Pathol 40: 996-1005, 2013.

3. Vishnu P, Chuda RR, Hwang DG and Aboulafia DM: Isolated granulocytic sarcoma of the nasopharynx: A case report and review of the literature. Int Med Case Rep J 7: 1-6, 2013.

4. Wang YF, Li Q, Xu WG, Xiao JY, Pang QS, Yang Q and Zhang YZ: Rare myeloid sarcoma/acute myeloid leukemia with adrenal mass after allogeneic mobilization peripheral blood stem cell transplantation. Cancer Biol Med 10: 232-235, 2013.

5. Nafil H, Tazi I and Mahmal L: Myeloid sarcoma developing in prexisting hydroxyurea-induced leg ulcer in a polycythemia vera patient. Case Rep Med 2013: 497593, 2013.

6. Byrd JC and Weiss RB: Recurrent granulocytic sarcoma. An unusual variation of acute myelogenous leukemia associated with 8;21 chromosomal translocation and blast expression of the neural cell adhesion molecule. Cancer 73: 2107-2112, 1994. 
7. He J, Zhu L, Ye X, Li L, Zhu J, Zhang J, Xie W, Shi J, Zheng W, Wei G, et al: Clinical characteristics and prognosis of nonleukemic myeloid sarcoma. Am J Med Sci 347: 434-438, 2014.

8. Brähler S, Thielen I, Schwabe H, Engels M, Kreuzer KA, Wolf J and Ansén S: Rapid remineralization of multiple disseminated bone lesions after high-dose cytarabine in a patient with isolated myeloid sarcoma. Eur J Haematol 92: 537-540, 2014.

9. Kohli S, Lee M and Marshall S: A case report on the progression of myeloid sarcoma to form multiple metastatic deposits without developing acute myeloid leukaemia. Case Rep Hematol 2015 $162154,2015$.

10. Pileri SA, Ascani S, Cox MC, Campidelli C, Bacci F, Piccioli M, Piccaluga PP, Agostinelli C, Asioli S, Novero D, et al: Myeloid sarcoma: clinico-pathologic, phenotypic and cytogenetic analysis of 92 adult patients. Leukemia 21: 340-350, 2006.

11. Audouin J, Comperat E, Le Tourneau A, Camilleri-Broët S, Adida C, Molina T and Diebold J: Myeloid sarcoma: Clinical and morphologic criteria useful for diagnosis. Int J Surg Pathol 11: 271-282, 2003

12. Ho T, Sedarat F, Rao N and Pullarkat ST: Diagnostic confusion resulting from CD56 expression by cutaneous myeloid sarcoma. Rare Tumors 1: e51, 2009.

13. Yilmaz AF, Saydam G, Sahin F and Baran Y: Granulocytic sarcoma: A systematic review. Am J Blood Res 3: 265-270, 2013.

14. Amador-Ortiz C, Hurley MY, Ghahramani GK, Frisch S, Klco JM, Lind AC, Nguyen TT, Hassan A, Kreisel FH and Frater JL: Use of classic and novel immunohistochemical markers in the diagnosis of cutaneous myeloid sarcoma. J Cutan Pathol 38: 945-953, 2011.

15. Vachhani P and Bose P: Isolated gastric myeloid sarcoma: A case report and review of the literature. Case Rep Hematol 2014 541807, 2014

16. Bain EE, Rothman I and Lin L: De novo myeloid sarcoma in a 4-month-old infant: A case report and review of the literature. J Cutan Pathol 40: 321-325, 2013.
17. Zhou J, Bell D and Medeiros LJ: Myeloid sarcoma of the head and neck region. Arch Pathol Lab Med 137: 1560-1568, 2013

18. Elyamany G, Khan M, El Hag I, El-Zimaity M, Albalawi M and Al Abdulaaly A: Generalized lymphadenopathy as the first presentation of granulocytic sarcoma: A diagnostic challenge. Case Rep Med 2013: 483291, 2013.

19. Raphael J, Valent A, Hanna C, Auger N, Casiraghi O, Ribrag V, De Botton S and Saada V: Myeloid sarcoma of the nasopharynx mimicking an aggressive lymphoma. Head Neck Pathol 8: 234-238, 2014.

20. Craig JW and Lin RJ: Paraneoplastic autoimmunity associated with testicular myeloid sarcoma and chronic myelomonocytic leukemia. Case Rep Hematol 2013: 656543, 2013.

21. Chimenti S, Fink-Puches R, Peris K, Pescarmona E, Pütz B, Kerl $\mathrm{H}$ and Cerroni L: Cutaneous involvement in lymphoblastic lymphoma. J Cutan Pathol 26: 379-385, 1999.

22. Brunning RD, Borowitz M, Matutes E, et al: Precursor B-cell and T-cell neoplasms. In: WHO Classification of Tumours. Pathology and Genetics of Tumours of Haematopoietic and Lymphoid Tissues. Jaffe ES, Harris NL, Stein H and Vardiman JW (eds). Vol. 3. IARC Press, Lyon, pp109-117, 2001.

23. Maitra A, McKenna RW, Weinberg AG, Schneider NR and Kroft SH: Precursor B-cell lymphoblastic lymphoma. A study of nine cases lacking blood and bone marrow involvement and review of the literature. Am J Clin Pathol 115: 868-875, 2001.

24. Shi Y and Wang E: Blastic plasmacytoid dendritic cell neoplasm: A clinicopathologic review. Arch Pathol Lab Med 138: 564-569, 2014.

25. Zeng Q, Yuan Y, Li P and Chen T: Spontaneous remission in patients with acute myeloid leukemia with $\mathrm{t}(8 ; 21)$ or cutaneous myeloid sarcoma: Two case reports and a review of the literature. Intern Med 52: 1227-1233, 2013. 